\title{
Rapidly Expanding Lung Abscess Caused by Legionella pneumophila in Immunocompromised Patients: A Report of Two Cases
}

\author{
Takayuki Mryara***, Kaori ToKaShiKI**, Tsutomu ShImoji**, Kazunori TAMAKI**, \\ Michio KoIDE* and Atsushi SAITO*
}

\begin{abstract}
We describe two cases of lung abscess caused by Legionella pneumophila in immunocompromised patients. The first case had been treated initially with $60 \mathrm{mg}$ prednisolone for ulcerative colitis, and $L$. pneumophila serogroup 1 was isolated from sputum samples after cavitation of the lung lesion. The second case was diagnosed as plasma cell lymphoma at post-mortem examination. L. pneumophila serogroup 5 was isolated from the contents of lung abscess, together with Enterococcus faecium and Prevotella intermedia in the post-mortem examination. Lung abscess caused by Legionella is unusual. Here, we discuss the difficulty of diagnosis of legionellosis in patients with unusual chest radiographic findings.
\end{abstract}

(Internal Medicine 41: 133-137, 2002)

Key words: legionellosis, pneumonia, oropharyngeal aspiration, chest radiogram, computed tomogram, urinary antigen detection

\section{Introduction}

Legionella is an important causative pathogen of bacterial pneumonia. Pneumonia caused by this pathogen is indistinguishable from that caused by other bacteria. Lung abscess caused by this organism is unusual and only a few cases have been reported in the literature (1-7). We encountered two cases of Legionella lung abscess with immunocompromised status. To our knowledge, only one case of Legionella lung abscess was reported in Japan previously (7). Here we discuss the difficulty of diagnosis of legionellosis in patients with unusual chest radiographic findings and on the possible role of oropharyngeal aspiration in the transmission of legionellosis.
Case Report

\section{Case 1}

A 30-year-old woman was admitted to the hospital for further management of ulcerative colitis. She was treated with 60 $\mathrm{mg}$ of oral prednisolone followed by gradual tapering of the dose. Twenty-seven days after commencement of this treatment, she developed Herpes zoster infection, which was successfully treated with aciclovir. Forty-five days after commencement of prednisolone therapy, when the dose was tapered to 30 $\mathrm{mg}$ per day, she developed fever of $38.8^{\circ} \mathrm{C}$. The patient complained of mild cough and left pleuritic pain. Laboratory examination showed a leukocyte count of $13,900 / \mathrm{mm}^{3}$ and CRP level was $9.49 \mathrm{mg} / \mathrm{dl}$. The chest radiograph showed a welldefined round opacity in the left upper lobe (Fig. 1) that had not been present on admission. Computed tomogram of the chest obtained on the following day revealed multiple areas of infiltration (Fig. 2). Two days after the chest radiogram shown in Fig. 1, the opacities were noted to have expanded rapidly (Fig. 3), which coincided clinically with the deterioration of dyspnea. On the same day, we obtained a specimen for determination of the causative microorganism by broncho-endoscopy but culture of the aspirated fluid showed no significant growth of organisms. Based on this finding, the administered antibiotic was switched from $2.0 \mathrm{~g}$ of piperacillin to $1.0 \mathrm{~g}$ of panipnem/betamipron. In spite of the treatment, the infiltrative shadow grew in size and a new cavity was recognized (Fig. 4). This was associated clinically with expectoration of purulent sputum. At that stage, sputum smears were negative for Gram stain but thin rods were identified in smears prepared by Gimenez stain. Further cultures of sputum samples on buffered charcoal yeast extract with alpha-ketoglutarate (BCYEalpha) agar plates resulted in the isolation of Legionella pneumophila serogroup 1 on two separate occasions. After confirmation of these colonies, erythromycin, levofloxacin and rifampicin were administered in combination for the treatment of the abscess caused by Legionella pneumophila serogroup 1.

From *the First Department of Internal Medicine, University of the Ryukyus, Okinawa and **Nakagami General Hospital, Okinawa

Received for publication May 24, 2001; Accepted for publication October 23, 2001

Reprint requests should be addressed to Dr. Takayuki Miyara, the First Department of Internal Medicine, University of the Ryukyus, 207 Uehara, Nishiharacho, Okinawa 903-0215 


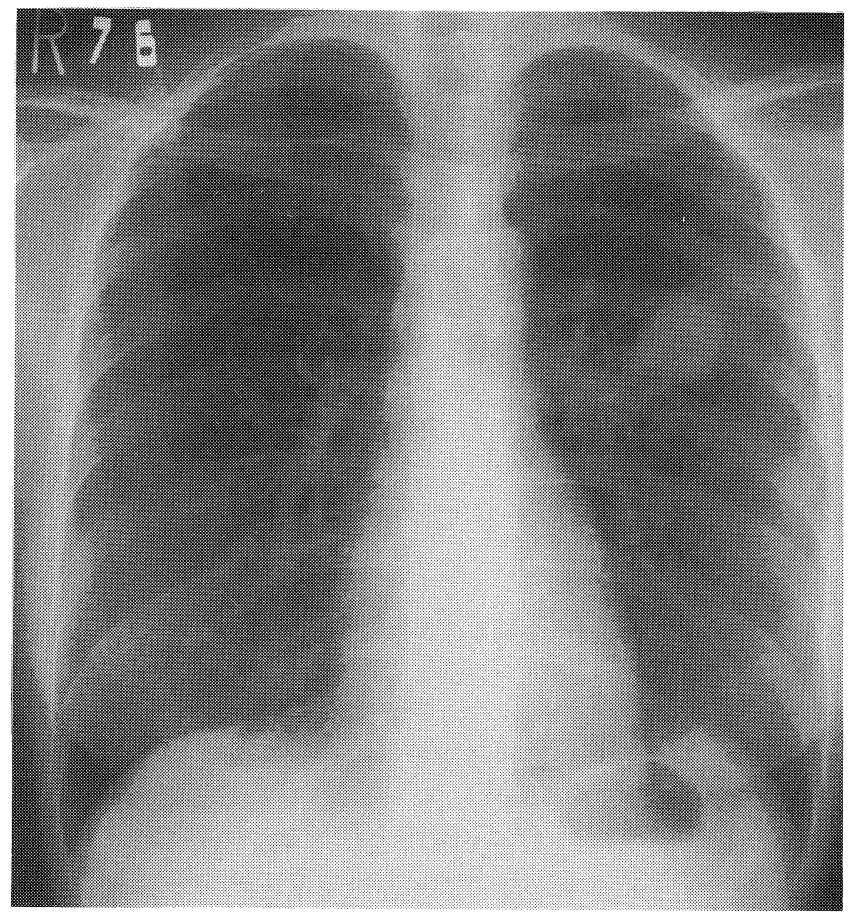

Figure 1. Case 1. Chest radiograph showing a well-defined round opacity with clear border in the left upper lobe.

Azithromycin and intravenous fluoroquinolone were not available at that time in Japan. Specific urinary antigen detection (Binax, Inc., Portland, Maine, USA) was also positive and serum anti-L. pneumophila serogroup 1 antibody titers were elevated from 1:32 to 1:256. After six weeks of treatment, these abscesses healed but multiple thin-wall cavities were noted on the chest radiograph (Fig. 5). Specific urinary antigen was detected for a period of three months. Although, oxygen saturation while breathing room air was maintained over $96 \%$ during the course of the disease, the patient was tachypneic (breathing rate: up to 30 per minute). Accordingly, supplemental oxygen therapy was provided at $3 \mathrm{l} / \mathrm{min}$.

\section{Case 2}

A 73-year-old man was admitted to our hospital for a oneweek history of persistent fever of $38^{\circ} \mathrm{C}$. He complained of easy fatigability, and physical examination showed generalized lymphadenopathy, splenomegaly and anemia. Laboratory tests showed a peripheral leukocyte count of $18,700 / \mathrm{mm}^{3}$, CRP of $10.8 \mathrm{mg} / \mathrm{dl}$, and hemoglobin of $9.7 \mathrm{~g} / \mathrm{dl}$. Pathological examination of a cervical lymph node biopsy showed features of reactive lymph node swelling. Bone marrow examination and surface marker analysis of lymphocytes were not informative and could not establish the diagnosis. Fever was persistent but repeated blood cultures were negative. Nine days after hospitalization, body temperature increased to $40^{\circ} \mathrm{C}$ and computed tomogram of the chest showed a round opacity in the left $\mathrm{S}^{3}$ (Fig. 6), which was not evident on the chest radiogram taken

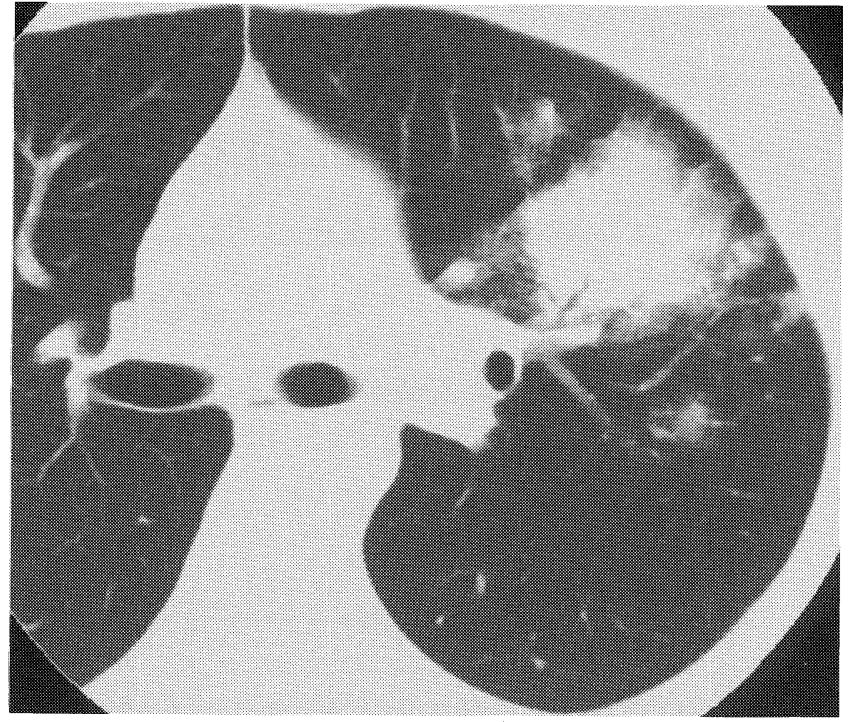

A

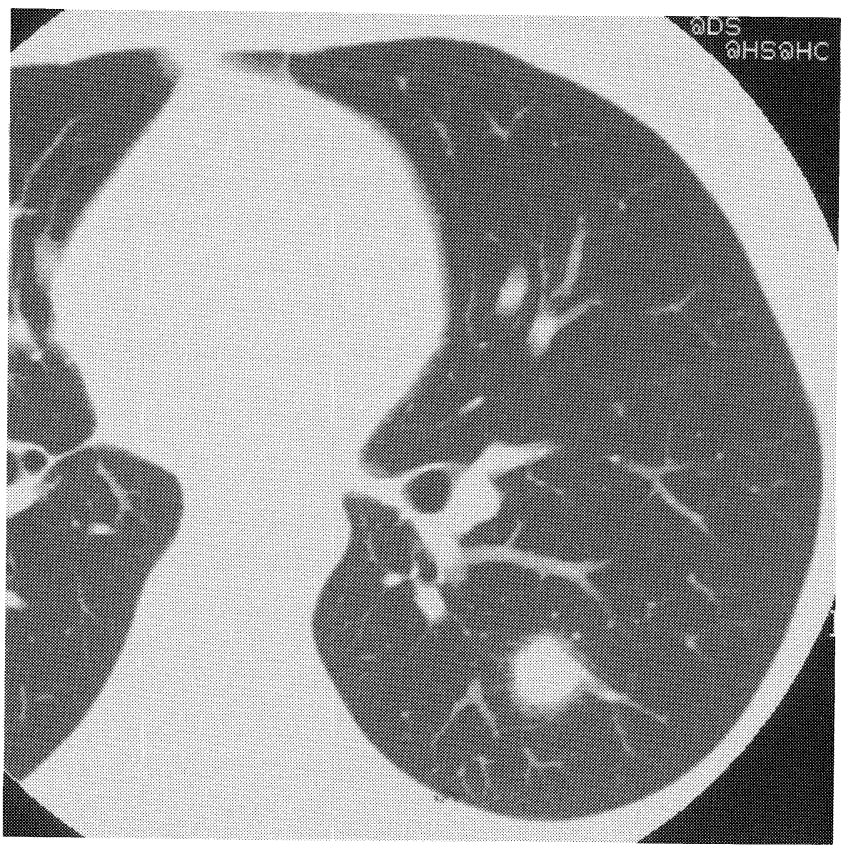

B

Figure 2. A, B. Case 1. Computed tomogram of the chest obtained on the day following the chest $\mathrm{X}$-ray shown in Fig. 1. Note the presence of multiple infiltrates.

on admission. Laboratory results at that stage showed peripheral leukocytosis $\left(23,300 / \mathrm{mm}^{3}\right)$ and elevated CRP $(20.9 \mathrm{mg} /$ dl). Arterial blood gas analysis while breathing room air showed $\mathrm{pH} ; 7.51, \mathrm{PaCO}_{2} ; 25, \mathrm{PaO}_{2} ; 64, \mathrm{HCO}^{3-} ; 25.2$ and $\mathrm{BE} ;-1.5$. In spite of administration of piperacillin and isepamicin following the recognition of the pulmonary infiltrate, the chest opacity expanded rapidly. To determine the causative organism, 


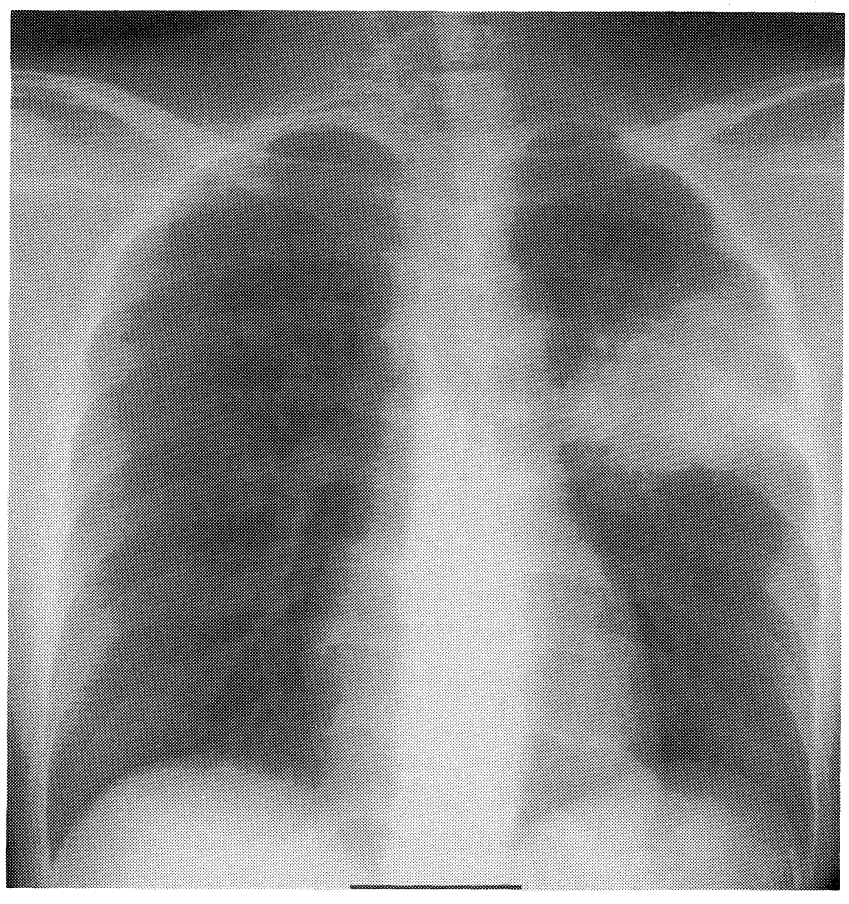

Figure 3. Case 1. Two days after the chest radiograph of Fig. 1. Note the rapid expansion of the round opacities.

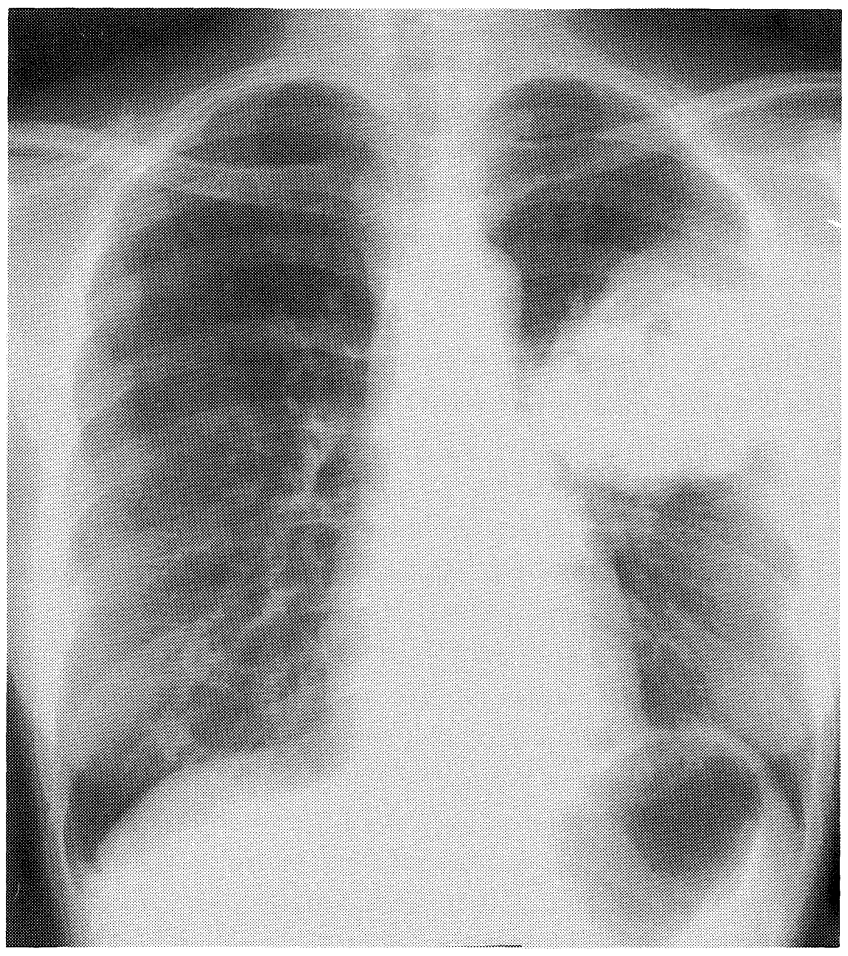

Figure 4. Case 1. Four days after the chest radiogram shown in Fig. 1. Note the presence of cavitation in the dense consolidation.

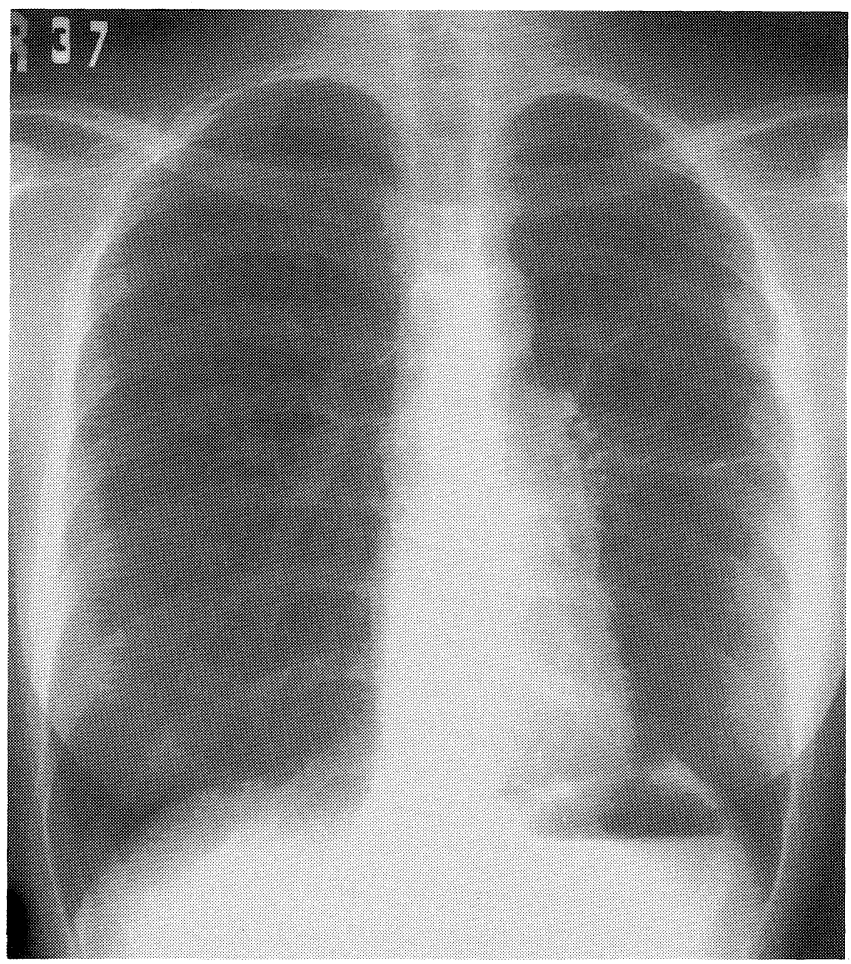

Figure 5. Case 1. Three months after the chest radiogram of Fig. 1. The abscesses healed but multiple thin-wall cavities were left.

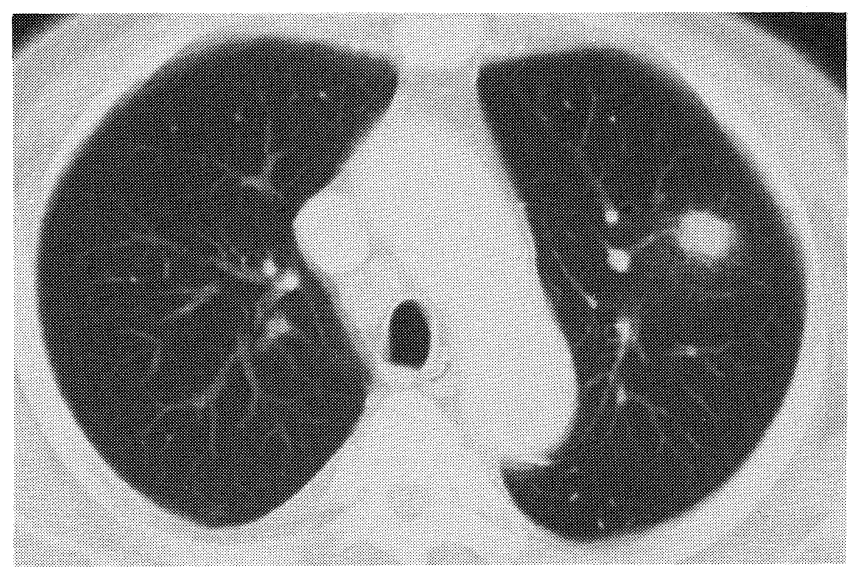

Figure 6. Case 2. Computed tomogram of the chest taken nine days after hospitalization. Note the presence of a round opacity in the left $S^{3}$, which was not evident on the chest radiogram taken few days earlier. 
broncho-endoscopic examination was performed on the $11^{\text {th }}$ day of hospitalization. However, no pathogenic organisms were isolated from the bronchial aspirate and Gimenez stain examination was negative. Three days after the first chest computed tomogram, the dense consolidation showed further expansion (Fig. 7A) and 10 days later, cavitation was subsequently noted

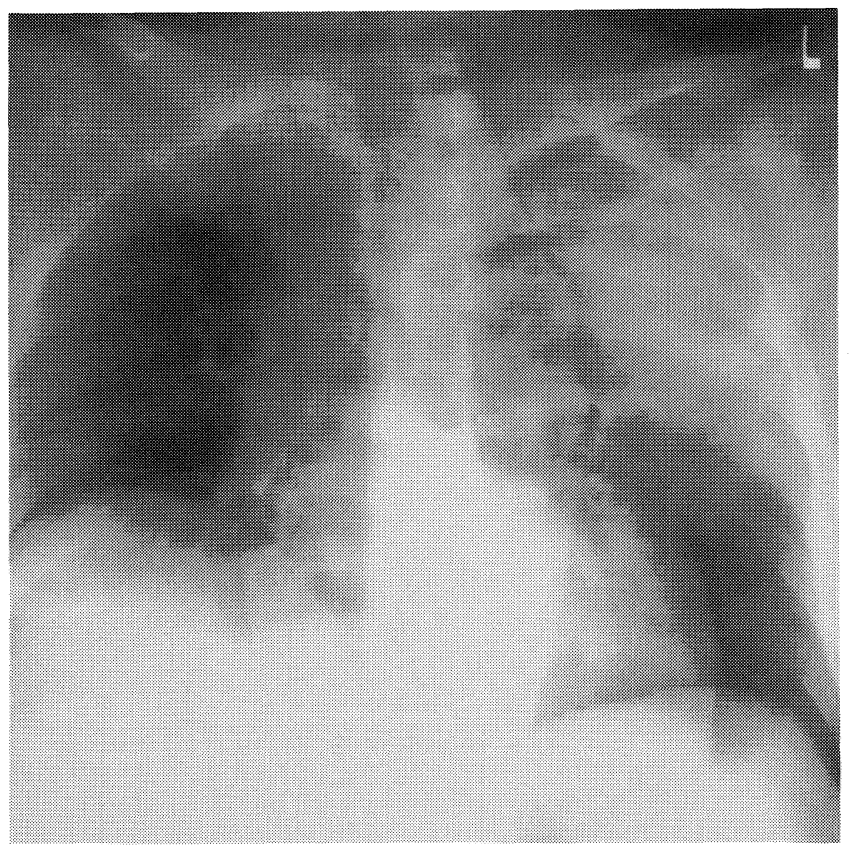

A

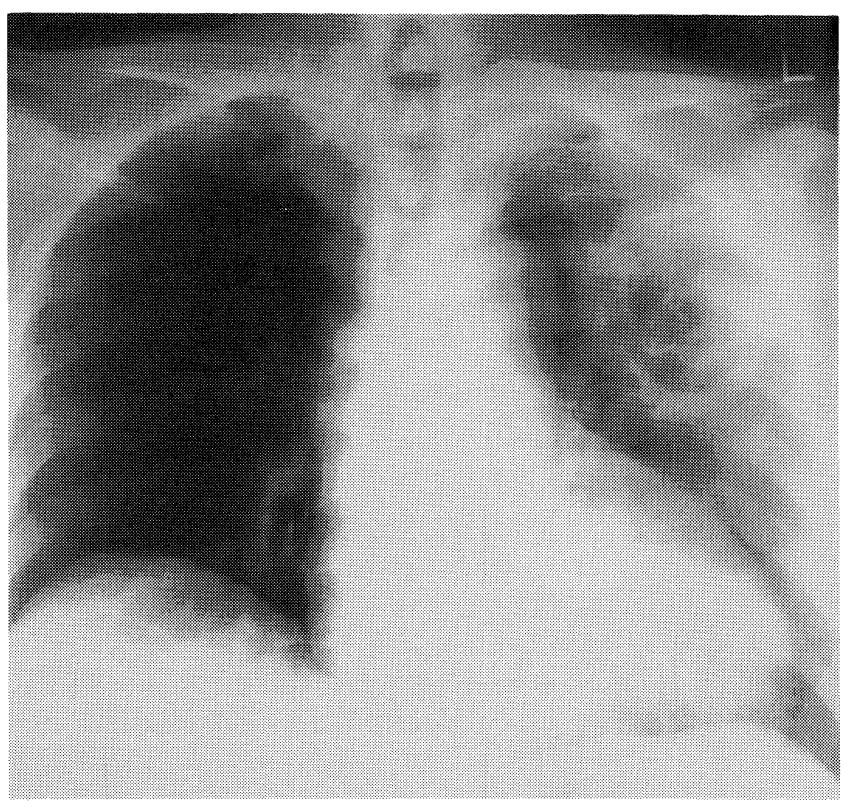

$\mathrm{B}$

Figure 7. A, B. Case 2. Chest radiograph showing rapid expansion of the dense consolidation $(A)$ and cavitation $(B)$. in the consolidation (Fig. 7B). On the $29^{\text {th }}$ day of hospitalization, the patient suddenly developed acute renal failure and died of cardiac arrhythmia. Post-mortem examination performed on the same day revealed that the pre-existing condition was plasma cell lymphoma. The content of lung abscess in the left upper lobe was aspirated under aseptic conditions. L. pneumophila serogroup 5, Enterococcus faecium and Prevotella intermedia were simultaneously isolated from the specimen. The patient had not used a shower for bathing due to the severe clinical condition, and no nasogastric tube was used during hospitalization.

\section{Discussion}

We describe two immunocompromised patients with lung abscess. The disease commenced as a nodular pulmonary consolidation without obvious interstitial change that expanded rapidly and finally developed into lung abscess. The diagnosis was established by isolation of L. pneumophila in both cases. The chest radiographic findings were unusual for Legionella pneumonia in Japan (7) therefore we did not suspect pneumonia caused by Legionella species until isolation of the organism. However, similar findings on the chest radiogram in patients with legionellosis have been described in Western countries mainly in immunocompromised patients (1-6) such as post-transplant patients $(2,3)$, patients on high-dose corticosteroid therapy $(1,4)$ and AIDS patients $(6)$. The causative pathogen in these reports was Legionella micdadei, formerly known as "Pittsburgh Pneumonia Agent". In Japan, only one case of lung abscess caused by Legionella dumoffii had been reported previously (7). The exact mechanism of transmission and frequency of lung abscess in legionellosis are not clear. However, from the description in western countries $(1-4,6)$, abscess formation is often detected in legionellosis in immunocompromised patients. Therefore, legionellosis should be considered in the differential diagnosis of a round opacity in the chest radiogram in febrile immunocompromised patients.

Legionella species is a facultative intracellular growth pathogen and it multiplies in phagocytosed macrophages, therefore, the main growth inhibition mechanism of the host defense against Legionella species is cell-mediated immunity (8). For this reason, immunocompromised status is one of the major risk factors of Legionella infection. In our patients, Case 1 received long-term corticosteroid therapy and Case 2 was diagnosed as plasma cell lymphoma, but both had almost intact non-immunospecific defense mechanism including neutrophils. Although the exact mechanism of abscess formation in the lung of immunocompromised patients by Legionella is not clear yet, it is possible that the number of Legionella is markedly increased in the infected area in such patients, but the host nonimmunospecific inflammatory reaction aimed at localization of the inflammation subsequently results in necrosis of the lung parenchyma and abscess formation in immunocompromised patients.

Although aerosolization is the widely accepted mode of transmission of Legionella, a number of investigators (9-12) 
suggested that oropharyngeal aspiration might be the mode of transmission in at least a proportion of patients with Legionella pneumonia. In Case 2, L. pneumophila serogroup 5 was isolated simultaneously with $E$. faecium and $P$. intermedia. This finding suggests that aspiration of oropharyngeal secretion was the sole mechanism of legionellosis. Furthermore, this finding also suggests that the bacteria in the oral cavity might synergistically enhance Legionella species-induced abscess formation.

Isolation of the microorganism is the golden standard for the correct diagnosis of legionellosis, therefore we attempted to isolate Legionella including staining of bronchial aspirates obtained by broncho-endoscopy by Gimenez staining, although this procedure failed to detect the causative microorganism. It is noteworthy that multiple pathogens were involved in the second case. To our knowledge, only a few cases have reported the identification of multiple pathogens in patients with legionellosis. Therefore, further studies of this type of infection and transmission through oropharyngeal aspiration are necessary.

\section{References}

1) Myerowitz RL, Pasculle AW, Dowling JN, et al. Opportunistic lung infection due to "Pittsburgh pneumonia agent". N Engl J Med 301: 953-
958, 1979.

2) Ellis AR, Mayers DL, Martone WJ, Mitchell BL, Atuk NO, Guerrant RL. Rapidly expanding pulmonary nodule caused by Pittsburgh pneumonia agent. JAMA 245: 1558-1559, 1981.

3) Pope TL, Armstrong P, Thompson R, Donowitz GR. Pittsburgh pneumonia agent: Chest film manifestations. Am J Roentgenol 138: 237-241, 1982.

4) Rudin JE, Wing EJ. A comparative study of Legionella micdadei and other nosocomial acquired pneumonia. Chest 86: 675-680, 1984.

5) Halberstam M, Isenberg HD, Hilton E. Abscess and empyema caused by Legionella micdadei. J Clin Microbiol 30: 512-513, 1992.

6) Johnson KM, Huseby JS. Lung abscess caused by Legionella micdadei. Chest 111: 252-253, 1997.

7) Fujita I, Tsuboi H, Ohotsuka M, et al. Legionella dumoffii and Legionella pneumophila serogroup 5 isolated from 2 cases of fulminant pneumonia. Kansenshogaku Zasshi 63: 801-810, 1989 (in Japanese with English Abstract).

8) Horwitz MA. Cell-mediated immunity in legionnaires' disease. J Clin Invest 71: 1686-1697, 1983.

9) Yu VL. Could aspiration be the major mode of transmission for Legionella? Am J Med 95: 13-15, 1993.

10) Blatte SP, Parkinson MD, Pace E, et al. Nosocomial Legionnaires' disease: Aspiration as a primary mode of disease acquisition. Am J Med 95: 16-22, 1993.

11) Johnson JT, Yu VL, Best MG, et al. Nosocomial legionellosis in surgical patients with head and neck cancer: Implications for epidemiological reservoir and mode of transmission. Lancet ii: 298-300, 1985.

12) Marrie TJ, Haldane D, MacDonald S, et al. Control of endemic nosocomial Legionnaires' disease by using sterile potable water for high risk patients. Epidemiol Infect 107: 591-605, 1991. 